\begin{abstract}
Trorse
PORTIONS OF THIS PEPORT ARE IIIETIRTE. has been reproduced from the best availab? ability.
\end{abstract}

\title{
RELATIONS BETWEEN THE SIMULTANEOUS AND SEQUENTIAL TRANSFER OF TWO NUCLEONS
}

G. R. Satchler

Oak Ridge Nationa 7 Laboratory Oak Ridge, TN 37830 , U.S.A.

\footnotetext{
to be published in
Proceedings of La Rábida International Surmer Sch.ool on Heavy Ion Collisions
La Rábida (Huelva) Spain June 7-19, 1982

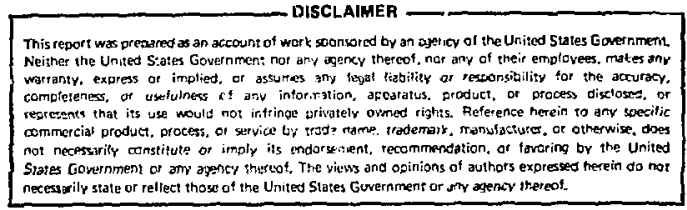

By acceptance of this article, the publisher or recipient acknowledges the U.S. Government's right to retain a nonexclusive, royal ty-free license in and to any copyright covering the article. 


\author{
G. R. Satchler \\ Dak Ridge National Laboratory \\ Oak Ridge, TN 37830, U.S.A.
}

\title{
1. Introduction
}

The transfer of two nucleons between projectile and target in a direct or peripheral reaction such as $(p, t)$ or $(160,14 \mathrm{C})$ may occur in one-step or two-steps. These we refer to as 'simultaneous' and 'sequential' transfers, respectively. In the former, the interaction acts once and both nucleons are transferred. In the latter, the interaction acts once to transfer one nucleon, the system then propagates in one or more intermediate states and is followed by a second action of the interaction to transfer the second nucleon. This process may be symbolized for the above examples as $(p, d ; d, \tau)$ and $\left({ }^{16} 0,15 \mathrm{~N} ;{ }^{15}, 1^{14} \mathrm{C}\right)$, implying the intermediate formation of a deuteron or the nucleus ${ }^{15} \mathrm{~N}$. (0f course, the intermediate system may exist in more than one state of excitation.)

In terms of a perturbation theory expansion, such as the distorted-wave Born series, simultaneous transfer is possible in first order while sequential transfer requires second order. This is illustrated in Fig. 1. We are accustomed, perhaps, to thinking that a first-order process is more likely than a second-order one. However, a closer examination can prepare us for the possibility that this nay not be so. The nuclear forces are predominantly two-body in character; hence, in first-order (Fig. 1a) only one of the two nucleons experiences an interaction. The possibility of finding that the other nucleon has also transferred arises only because its state within the projectile is not orthogonal to the state in the target into winich it transfers. This process corresponds to a kind of quantum-mechanical tunneling from the projectile to the target.1) However, in the two-step process (Fig. Ib) each nucleon is transferred under the direct influence of an interaction with the target; intuitively, this might seem more plausible. It requires an explicit calculation to determine which process is most likely in a given case, and such calculations are often beset with uncertainties. Nonetheless, it seems clear that the one-step and two-step amplitudes are frequently comparable in magnitude for light-ion reactions, 2 ) while the two-step may dominate in reactions with heavy ions. ${ }^{3)}$ (The existence of strong Q-window effects, especially with heavy ions, 4 ) may enhance the sequential process when there is a large mismatch between the entrance and exit channels. The gap may be bridged more easily in two steps, with the interaction acting twice.) Consequently, it is not safe to ignore the existence of sequential transfer. However, one of the min reasons for studying twonucleon transfers is to learn about the two-nucleon overlaps (existence and extent of pairing correlations, etc.). So we wish to know if this information is still 


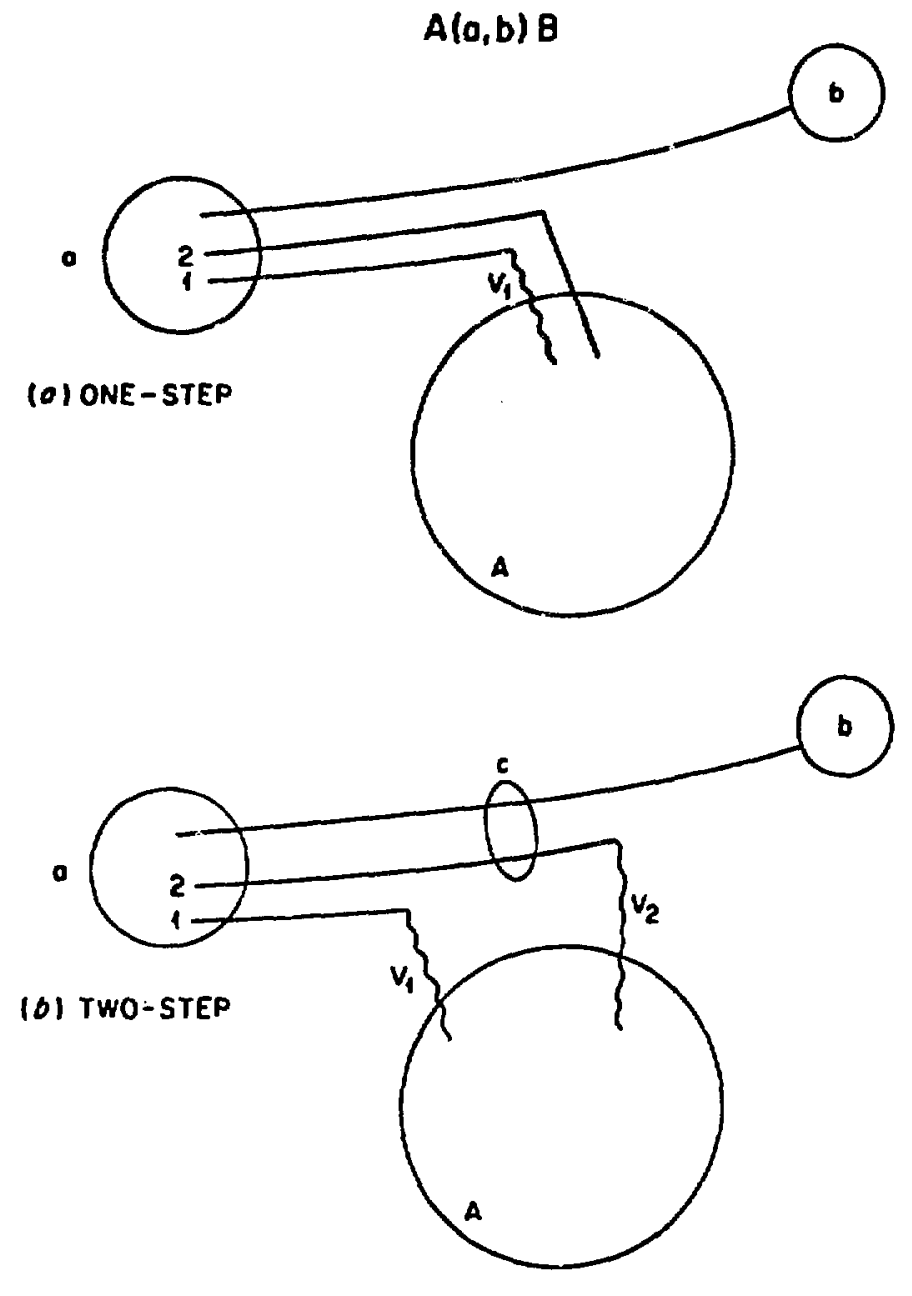

Fig. 1. (a) Simultanec - transfer, (b) sequential transfer. The wavy ine corresponds to one action of the projectile-target interaction. available when the reaction proceeds by two, sequential, onenucleon transfers. Our purpose here is to gain some insight into the relationship between the two amplitudes by using a simple approximate form of the theory. For simplicity, we shall discuss a light-ion reaction and, to be specific, we choose the $(t, p)$ reaction (or the inverse $(p, t)$ reaction). Similar considerations apply to other reactions, and we include some remarks concerning $(\alpha, d)$ reactions. This work (done in collaboration with W. T. Pinkston) is described in a paper shortly to be published in Nuclear Physics A. Hence, here we shall only give an abbreviated and schematic outline of the theory, leaving the reader to consult the forthcoming paper for the details.

\section{The $(t, p)$ or $(p, t)$ Reaction}

Apparently contradictory evidence exists for the relative importance of the two-step or sequential-transfer mechanism in $(t, p)$ or $(p, t)$ reactions. Indeed, the results of a confusing variety of sophisticated calculations are available, each of which treats properly some, but not all, aspects of these transitions. Among these aspects are: (i) the use of a realistic triton wavefunction and the associated realistic interaction;5-8) (ii) an exact treatment of the finite-range of the interactions (as opposed to use of a zero-range approximation), in both the onestep $^{5-9}$ and the two-step ${ }^{8-11)}$ amplitudes; (iii) accounting for the nonorthogonality correction to the two-step term, $2,9,11,12)$ which tends to cancel the one-step amplitude;12-14) (iv) allowing the intermediate n-p system of the two-step process to exist in continuum states, both spin-singlet and triplet, as well as in the bound, triplei deuteron ground statel2,15) (sometimes this is called the 'deuteron break-up effect'!. In addition, there is a sensitivity of the results to the optical potentials employed, and a particular sensitivity of the cross section 
magnitudes to the nuclear wavefunctions used to construct the two-nucleon overlaps.6,7)

In view of the complexity of the theoretical description of this transfer process, the agreement with measured cross sections obtained by calculations wich only include some of these aspects must be viewed with some caution. In this situation it is valuable to have an understanding of any general, albeit approximate, features that the amplitudes may possess, independent of these details, which may illuminate the results of more detailed calculations.

The central point of the present study is to use a closure approximation for the intermediate nuclear states that appear in the second-order distorted-wave-Born expression for the two-step amplitude in order fo show that the simultaneous and sequential processes tend to depend in the same way upon the nuclear structure of the initial and final states ${ }^{3,16)}$ and to exhibit similar selection rules. Various additional simplifying assumptions, such as zero-range, can be used to demonstrate more dramatically17) the basic similarity of the two amplitudes in a way that is not obscured by partial-wave expansions, angular momentum algebra, etc. ${ }^{3}$ ) While these may have large effects upon the magritude of the amplitudes, they do not affect the underlying structure. They do help to explain why the sequential and simultaneous processes tend to yield similar angular distributions.

A closer examination of the spin angular momenta involved modifies the initial conclusion that the selection rules are the same in both cases. For example, in the two-neutron transfer $(t, p)$ reaction, if the $n-p$ system or 'deuteron' associated with the intermediate states of the two-step amplitude appears in spin-singlet or spin-triplet states with equal weight, the selection rules for one- nd two-step are identical. However, constraining it to be in a triplet state criy (such as the common assumption that it is the physical deuteron ground state) acts as a spinfilter and determines that the two neutrons are transferred with a particular mixture of total spin $S=0$ or 1 . This mixture will differ from that for the one-step term. In this way, inclusion of singlet deuterons might change the results for vector analyzing powers and might modify conclusions that have been drawn from measurements of these quantities.18)

\subsection{First-and Second-Distorted-Waves Born Approximations}

We do not refer explicitly to spins for the schematic presentation given here; see Ref. 17 for details. Consider the $A(t, p) B$ reaction, so that $B=A+2 n$. The first-order (or simultaneous transfer) amplitude has the form ${ }^{19}$ )

$$
I^{(T)}=\int x_{p}^{(-)^{*}}\left(\mathbb{R}_{p}\right)(B \mid A)\left(V_{1}+V_{2}\right) \psi_{t} x_{t}^{(+)}\left(\mathbb{R}_{t}\right)
$$

where the $x_{p}$ and $x_{t}$ are the usual distorted waves describing the centre-of-mass motion of the proton and triton, while $V_{1}$ and $V_{2}$ are the residual interactions 
acting on neutrons 1 and 2 , and $\psi_{t}$ describes the internal state of the triton. $A l$ so, $(B \mid A)$ is the overlap between the initial and final nuclear states,

$$
(B \mid A) \equiv \int \psi_{B}^{\star}\left(\underline{r}_{1}, \underline{\sim}_{2}, \xi\right) \psi_{A}(\xi) d \xi=\Phi^{B A}\left(\underline{\sim}_{1}, r_{2}\right) \text {, }
$$

say. All the nuclear structure information, including pairing correlations, is manifest through this $\Phi^{B A}$, which is often called the two-nucleon form factor for the transition.

The second-order (or sequential transfer) amplitude for the processes $A+t+C+d+B+p$ has the form $\left.{ }^{19}\right)$

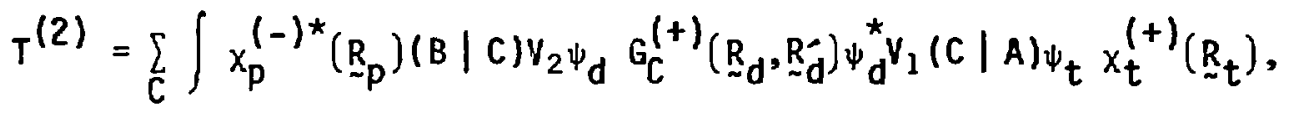

where $\psi_{d}$ is the internal state of the intermediate deuteron and $G_{C}$ is the Green function describing the relative motion (in the Coulomb + optical potential) of the intermediate $d+C$ system. For exampie, if we ignored the optical potential and the Coulomb field so that the system propagated in plane waves with momentum $k_{d}$, we would have

$$
G_{C}\left(\underset{\sim}{R}, \underset{\sim}{R^{-}}\right)+\exp \left(i k_{d} s\right) / s, \quad s=\left|\underset{\sim}{R}-R_{\sim}^{-}\right|
$$

The wavenumber $k_{d}$ could be re-interpreted as the local value $k_{d}(\bar{R})$ at the position $\bar{R}=\frac{1}{2}\left|R+R^{-}\right|$in the potential, thus introducing a 'local energy approximation' 3 ) for the effects of the optical and Coulomb potentials.

\subsection{Closure for the Two-Step Amplitude}

Now we do not see a two-nucleon overlap appearing in the two-step amplitude (3), but a sum of products of one-nucleon overlaps weighted by $G_{C}$, where

$$
(C \mid A)=\int \psi_{C}^{*}\left(r_{l}, \xi\right) \Psi_{A}(\xi) d \xi=\phi^{C A}\left(\underline{r}_{1}\right) \text {. }
$$

say, with a similar expression for $(B \mid C)$. The question is, "Is the information on two-neutron correlations that is carried by $\Phi^{B}\left(r_{1}, r_{2}\right)$ in the first-order $T^{(1)}$ lost if it should happen that the second-order $\mathrm{T}^{(2)}$ is important?". The answer is "No" if the important intermediate states $r$ are sufficiently close in energy that the propagators $G_{C}$ do not vary much. For then we may replace each $G_{C}$ by an average value $\bar{G}_{C}$ and then use the closure property of a complete set of states,

$$
\left.\sum \mid c\right)(C \mid=1
$$

to collapse the sum over $C$ in $E q .(3)$ :

$$
\sum_{C}(B \mid C) G_{C}(C \mid A) \approx G_{C} \sum_{C}(B \mid C)(C \mid A)=G_{C}(B \mid A) \text {. }
$$

The result is the appearance of the same two-neutron overlap as that which occurs in the one-step amplitude $T^{(1)}$. Consequently, when the approximation ( 7$)$, is 
sufficiently good, nuclear structure effects such as pairing correlations will manifest themselves in much the same way in one-step or two-step processes. In this way, one can understand how analyses of measured cross sections, using the theory for a one-step transfer alone, may yield useful information on the relative behavior of spectroscopic factors for different states and different nuclei,20) even when sequential transfer may be important.

Althosgh it is not necessary for the present argument, it is instructive to introduce further the usual zero-range approximation 19 ) for the one-step process,

$$
V_{\psi_{t}} \approx D(r) \delta(\rho)
$$

where $r=\left|r_{1}-r_{2}\right|$ is the separation of the two neutrons, while 2 is the position of the proton relative to the centre of mass of the two neutrons. We also take the usual zero-range approximation19) for each of the one-neutron transfers in the twostep process. Then the amplitudes (1) and (3), with the closure (7), become

$$
\begin{aligned}
& T^{(1)} \approx \int x_{p}^{(-)^{*}}\left(\underset{\sim}{\left.R^{-}\right) \phi^{B A}}\left(\underset{\sim}{r_{1}}, \underset{\sim}{r_{2}}\right) D(r) x_{t}^{(+)} \underset{\sim}{(R)}\right) \underset{\sim}{d} \underset{\sim}{d R}, \\
& T^{(2)} \approx D_{0}(d, p) D_{0}(t, d) \int x_{p}^{(-)^{*}}\left(\underline{\sim}^{-}\right) \Phi^{B A}\left(\underline{r}_{1}, \underline{\sim}_{2}\right) G\left(\underline{r}_{1}, \underline{\sim}_{2}^{-}\right) x_{t}^{(+)}(\underset{\sim}{R}) d \underset{\sim}{r} d R,
\end{aligned}
$$

where $\underline{\sim}_{2}^{\prime}=\underline{\sim}_{2} A /(A+1),{\underset{\sim}{R}}^{-}=\underset{\sim}{R A} /(A+2)$ and $\underset{\sim}{R}=\frac{1}{2}\left(\underline{r}_{1}+\underline{r}_{2}\right)$. The $D_{0}$ are the usual zerorange normalization constants. There is a remarkable similarity of structure in the two amplitudes, which differ only by the appearance of the short-ranged overlap function $D(r)$ in $T^{(1)}$ and the average Green function $G$ in $T^{(2)}$. In the plane-wave approximation (4), $G$ is also a function only of $r$ (if $A \gg 1$ so we can neglect the small recoil correction). (Of course. is complex, whereas $D$ is usually taken to be real.) Consequently, we should not l. surprised to find that one-step and twostep transfers have rather similar angular distributions.

\subsection{Further Comments on the Closure Approximation}

One can easily construct model situations in which the procedure (7) beccmes exact. For example, consider two-neutron transfer to a closed-shell target in which the intermediate states $\psi_{C}$ are pure single-particle j-states and the residual state $\psi_{B}$ has a pure $j^{2}$ configuration. Only one intermediate state can contribute, namely the corresponding single-particle state in nucleus $A+1$ with the same $j$, and Eq. (7) becomes exact.

The expression (3) assumes a single intermediate state for the intermediate n-p system, in this case the ground state of the deuteron. More generally, both inter: mediate nuclei may be in more than one state and expressions like (3) need to be supplemented by an additional sum over the states of the "deuteron". In a heavyion reaction, the intermediate nuclei may be comparable and hence should be treated on the same footing. This was done by Feng, et al.3) when they applied the closure approximation. The tendency has been to treat light-ion reactions differently, by 
assuming that the intermediate light particle remains in its ground state. This has been a matter of expediency; excited states of light ions are unbound and thus introduce a continuum of intermediate $\geq 3$-body states which greatly increases the complexity of the calculation. This has been investigated 2,25$)$ for the intermediate 'deuteron' in $(p, t)$ reactions.

The closure approximation (7) may be applied to the states of $c$, as well as those of $C$, in the process $A+a+C+C+B+a$, if the important contributions still come from a sufficiently narrow band of energies (i.e. narrow enough that the Green function $G_{C_{c}}$ does not vary much). This results in the appearance of a second twonucleon overlap or form factor ( $b \mid a)$, which again is the same as that which occurs in the one-step amplitude $T^{(1)}$. Then the nature of the intermediate nucleus $c$ plays no rôle and, for example, angular nomentum selection rules are the same for one-step and two-step transfer. It is plausible that this is approximately valid for heavy-ion reactions ${ }^{3}$ ) where the important intermediate states are likely to be bound states of moderate excitation energy. It is not so obvious for light-ion reactions. The explicit calculations 12,15$)$ for ${ }^{48} \mathrm{Ca}(\mathrm{p}, \mathrm{t})$ at 20 and $40 \mathrm{MeV}$ included the $n-p$ scattering states, both singlet and triplet. Important contributions came from the continuum states at $40 \mathrm{MeV}$, but the effect (on the cross section) was small at $20 \mathrm{MeV}$. The intermediate spin-singlet $n-p$ state is always associated with continuum states of relative motion. Hence, the transition would be dominated by triplet intermediate states if the contribution of the continuum were negligible. This constitutes a spin-filter through which the two-step process must pass and introduces differences in the spin selection rules compared to the one-step process. In the simpiest triton wavefunction, 21 ) the two neutron spins are coupled to $S=0$ and the triton spin $-\frac{1}{2}$ is due to the odd proton. Then we have $S=0$ transfer in the one-step amplitude. After recoupling the spins within the triton, we find a linear combination of $s_{d}=0$ and 1 for the proton plus one of the neutrons. The assumption of an intermediate triplet deuteron in the two-step process selects $s_{d}=1$; i.e. it filters out the $s_{d}=0$ component. The resulting $n+n+p$ function is no longer pure $S=0$; it also contains $S=1$ components for the two neutrons. However, if the singlet and triplet deuteron states were included with equal weights, the filter is removed and only $S=0$ transfer is possible for the sequential transfer also.

\section{The $(\alpha, d)$ Reaction}

A similar analysis has been made of the $(\alpha, d)$ reaction. 17) Two sequential processes are ayailable here, $(\alpha, t ; t, d)$ and $(\alpha, h ; h, d)$ (where $h \equiv{ }^{3}$ He). Only $s_{d}=1$ transfer is possible for the simultaneous transfer because of the zero spin of the alpha. If we assume an S-state of maximum symmetry for the alpha, oniy spin $-\frac{1}{2}$ states are allowed for the triton and helion. Thus, although they act as spin $-\frac{1}{2}$ 'spin filters', they impose no further constraint on the spin transfer. The spintransfer selection rules are the same for simultaneous and sequential transfer. 
There could be, however, an isospin filtering effect which would result from any difference in propagation of the $t$ and $h$ particles in unsymmetric, charged nuclear matter (i.e. if they had different Green functions).

By introducing similar zero-range and closure approximations, the one-step and two-step amplitudes are reduced to the same forms as Eqs. (8) and (9) for the $(t, p)$ reaction. Again, the two amplitudes are seen to depend upon nuclear structure in the same way. The same two-nucleon overlap, analogous to Eq. (2), appears in both. In particular, there does not appear aniy phase difference between them that depends upon the spin of the residual nuclear state.

The latter point is especially relevant because of some observations of the Pittsburgh group $\left.{ }^{22}\right)$ on the ${ }^{208} \mathrm{~Pb}(\alpha, d)^{2} 10 \mathrm{Bi}$ reaction. Consider excitation of the $\left(g_{g / 2} h_{g / 2}\right)_{j}$ multiplet of states with $J=0,1, \ldots g$. Simple angular momentum considerations, together with the tendency of $(\alpha, d)$ reactions to favor large L-transfers, lead to the prediction that the one-step cross sections should show a regular stepped pattern when plotted against $J$, with the rise a $\dot{\tau}$ each step increasing as $J$ increases. DWBA calculations 22 ) confirm this (see Fig. 2a). The discussion of the present paper suggests that a similar pattern should be seen for the two-step process by itself and again explicit calculations 22 ) confirm this (Fig. 2a).

The measured cross sections ${ }^{22}$ ) show a rather different, saw-tooth pattern (Fig. 2). The predicted steady rise with $L$ transfer is observed; however, the cross sections for the even-J members of the $J=(L-1, L)$ pairs are larger than those for the odd-J members. Coupled-channels calculations ${ }^{22}$ ) including direct and sequential transfers reproduced these trends and seemed to indicate that the saw-tooth pattern resulted f:- ; successively constructive and destructive interferences of direct and sequential $\geq$ plitudes due to a $\mathrm{J}$-dependence in their relative phase. No such $\dot{j}-$ dependent phase factor appears in the present treatment ${ }^{\dagger}$, although this treatment is able to azcount for the trends shown by the individual one-step and two-step amplitudes in terms of the reaction dynamics and the LS-jj transformation coefficients involved in the two-nucleon overlap factor.17) Indeed, the integrand of the two-step amplitude differs from that for the one-step only by the replacement of the light-ion overlap function $D(r)$ by $\overline{\bar{G}}_{0}(r, R)$, the average of the triton and helion Green functions.17) The symmetric part of the two-nucleon overlap $\Phi\left(r_{2}, r_{1}\right)$, from which any $\mathrm{J}$-dependence must arise, is common to both amplitudes.

Our analysis makes it difficult for us to understand either the calculations presented in Refs. 22 or the experimental data, which seem to be nicely explained by the coupled-channels calculations. Cross sections for exciting the same states by $208 \mathrm{pb}\left({ }^{3} \mathrm{He}, \mathrm{p}\right){ }^{210} \mathrm{Bi}$ show a similar behavior. $\left.{ }^{23}\right)$ The $\left({ }^{3} \mathrm{He}, \mathrm{p}\right)$ reaction differs from $(\alpha, d)$ in that $S=1$ and $S=0$ transfer are both possible. However, nuclear structure

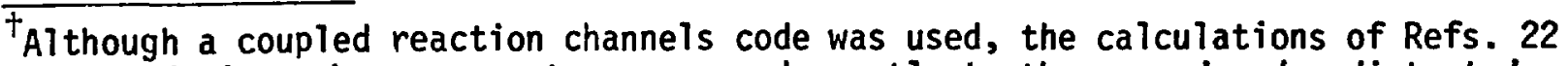
were made in such a way as to correspond exactly to the second-order distortedwave Born approximation discussed here, including the use of the zero-range approximation.
} 


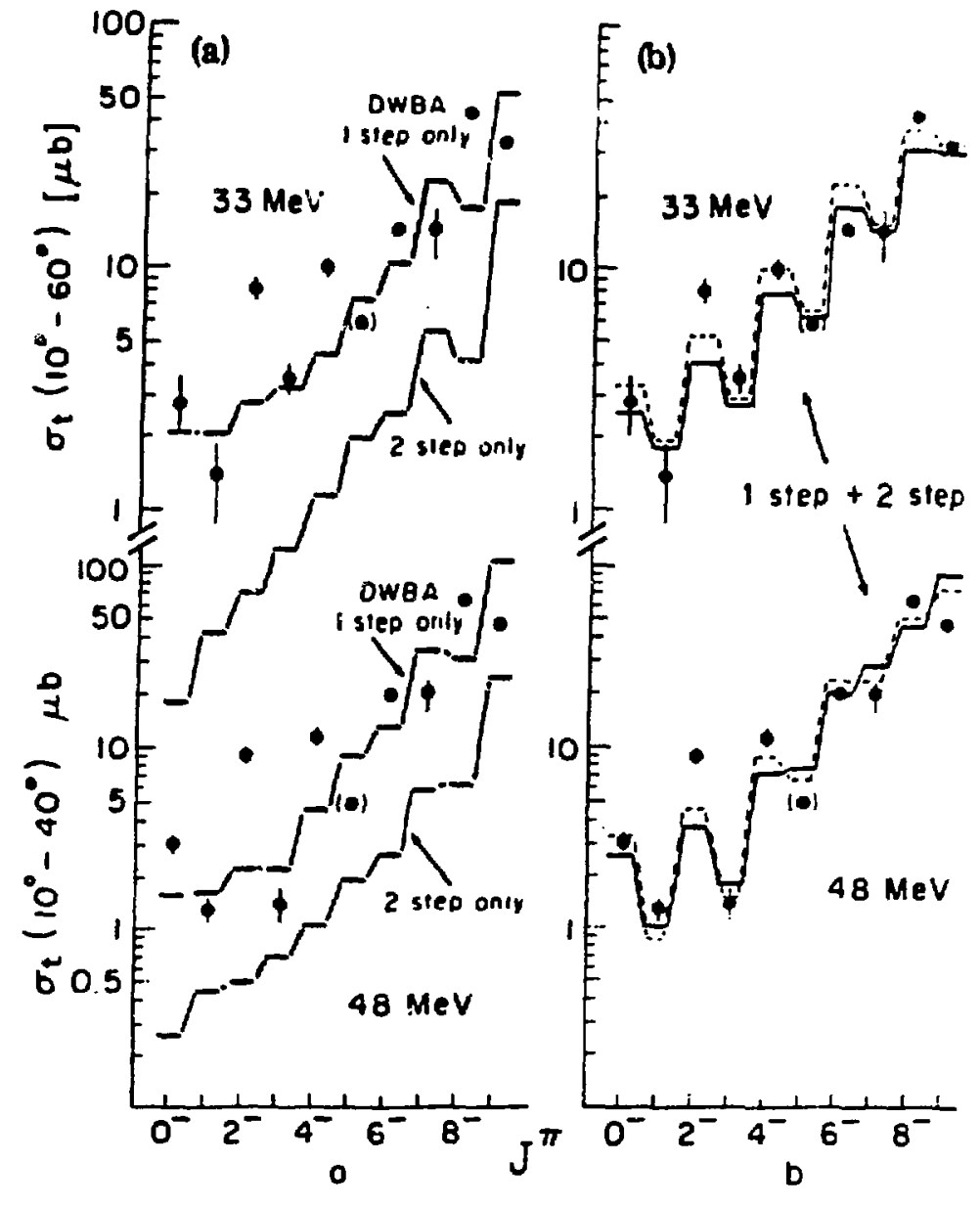

Fig. 2. Integrated cross sections, versus 3 , for the $208 \mathrm{~Pb}(\alpha, d)^{210} \mathrm{Bi}$ reaction exciting the

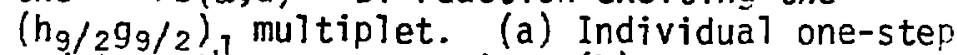
and two-step cross section; (b) coherent sum of one-step and two-step; as reported in Ref. 22. factors make the $S=0$ contribution much smaller than that for $S=1$, so that, except for kinematic effects, one sinould expect the two reactions to behave in a very similar way. Our approach differs from that using coupled channels in that we do not consider intermediate states explicit$1 y$ but make the closure approximation. However, with the assumption of a single $\left(g_{9 / 2} h_{g / 2}\right)$ configuration for the final state in the $208 \mathrm{~Pb}(\alpha, d)^{210 B i}$ case, the result of making the closure approximation yields the same result as restricting the intermediate states to be the $209 \mathrm{~Pb}$ and $209 \mathrm{Bi}$ ground states, as was done in the coupled-channels calculations. 221

\section{Some Remarks on Non-Orthogonality and Second-Order Processes}

In a rearrangement collision $A(a, b) B$, in which $(b, B)$ are a pair of nuclei for partition) different from $(a, A)$, the internal states in the initial and final channels are not orthogonal;19) for example,

$$
(b, B \mid a, A) \equiv \int \psi_{b}^{*}\left(\xi_{b}\right) \psi_{B}^{*}\left(\xi_{B}\right) \psi_{a}\left(\xi_{a}\right) \psi_{A}\left(\xi_{A}\right) d \xi_{a} d \xi_{A} \neq 0
$$

Since $\xi_{B}=\xi_{B}\left(\xi_{a}, \xi_{A}, \sim_{a A}\right)$, etc., this overlap remains a function of the a+A separation ${\underset{\sim a A}{\mathrm{a}}}$. Such overlaps vanish asymptotically $\left(r_{a A}+\infty\right)$, but remain finite in the region where the two nuclei overlap. This is just the region that contributes to transition amplitudes like (1) or (3). As a consequence, in general $\mathrm{Eq}$. (3) is not the correct second-order Bcrn approximation to the transition amplitude; there -is a "non-orthogonality (NO) correction" term. The expression for the NO term $^{14,19)}$ depends upon the particular choice, post or prior, made for the interactions in each of the two steps in the amplitude $T$ (2) of Eq. (3). If prior is chosen for the $a+A \rightarrow c+C$ step and post for the $c+C+b+B$ step, the $N$ term 
vanishes. For other choices it does not. For example, frequently post is chosen for both steps for sequential stripping (like the $(t, p)$ reaction) induced by light ions because then a zero-range approximation can be invoked for both of the steps (as was done in deriving Eq. (9)). Then the NO term has the form

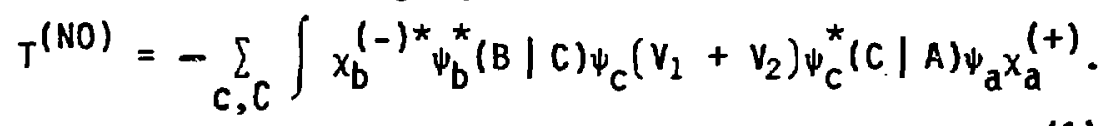

This has a structure intermediate jetween that for $T^{(1)}$ and $T^{(2)}$ of Eqs. (1) and (3); but note the overall minus sign. Indeed, if we sum over a complete set of states of both nuclei $c+C$ using Eq. (6) and its analogue for nucleus $c$, then $T^{\text {(NO) }}$ becomes $^{13)}$ equal and opposite to the one-step amplitude $T^{(1)}$. The two-step term $T^{(2)}$ (which should be summed over the same complete set of intermediate states) is then left as the lowest-order contribution to the transition. In practice, however, it is not feasible to use a complete set. Formally, it implies nonconvergence of the Born series because of the mathematical difficulties associated with the 3- and more-body states that appear when the intermediate states are unbound. One standard approach 19 ) is to regard our calculations as being done within a truncated model space, limited to 2-body channels, and with effective interactions. Thus, a complete set is ruled out from the start. Nonetheless, the tendency remains for $T^{(N O)}$ and $T^{(1)}$ to interfere destructively, sometimes quite strongly (see Refs. 9 and 12-15 for examples), and this enhances the importance of the two-step process described by $T^{(2)}$.

One final note: the separation of the second-order amplitude into $T^{(2)}$ and $T^{(N O)}$ is artificial. Despite the intuitive appeal of interpreting $T^{(2)}$ as "the two-step process", one cannot physically distinguish these two terms from each other (nor they from the first-order term $T^{(1)}$ ). This is emphasized by the lack of uniqueness in the separation into two parts; for example, if we had chosen the prior-post interaction form for $T^{(2)}$, there would be no $T^{(N O)}$ term and hence no possibility of the cancellation of $T^{(1)}$. This lack of uniqueness also renders ambiguous even the conceptual division into one-step and two-step. Only the total amplitude has direct physical meaning; its breakdown into pieces is merely a consequence of the way we do calculations. Nonetheless, the discussion given earlier can be valuable as an aid to understanding the results of those calculations. of course, the division within any particular calculation is (or should be!) unambiguous. Many calculations have ignored the possibility of No corrections (for example, the $(\alpha, d)$ calculations referred to earlier 22), usually as a matter of computational convenience. However, the closure argument given above, albeit invalid, remains as a warning that No effects may not be negligible, and the results of explicit calculations $\left.{ }^{9}, 12-15\right)$ support this conclusion. 
* Research sponsored by the Division of Basic Energy Sciences, U.S. Department of Energy, under contract W-7405-eng-26 with the Union Carbide Corporation.

1. I am indebted to R. M. Drisko for this remark.

2. For example, see N. Hashimoto and M. Kawai, Prog. Theor. Phys. 59, 1245 (1978).

3. For example, see D. H. Feng, T. Udagawa and T. Tamura, Nucl. Phys. A274, 262 (1976).

4. For example, see R. Bass, Nuclear Reactions with Heavy Ions, SpringerVerlag, Berlin, 1980 .

5. M. A. Nagarajan, M. R. Strayer and M. F. Werby, Phys. Lett. 68B, 421 (1977).

6. T. Takemasa, T. Tamura and T. Udagawa, Nuc1. Phys. A321, 269 (1979).

7. D. H. Feng, M. A. Nagarajan, M. R. Strayer, M. Vallieres and W. T. Pinkston, Phys. Rev. Lett. 44, 1037 (1980).

8. P. P. Tung, K. A. Erb, M. W. Sachs, G. B. Sherwood, R. J. Ascuitto and D. A. Bromley, Phys. Rev. C 18, 1663 (1978).

9. M. Igarashi and K. I. Kubo, Phys. Rev. C 25, 2144 (1982).

10. L. A. Chariton, Phys. Rev. C 14, 506 (1976).

11. N. Hashimoto, Progr. Theor. Phys. 59, 1562 (1978).

12. N. Hashimoto, Progr. Theor. Phys. 63, 858 (1980).

13. P. D. Kunz and E. Rost, Phys. Lett. 47B, 136 (1973).

14. T. Udagawa, H. H. Wolter ano' . R. Coker, Phys. Rev. Lett. 31, 1507 (1973);

U. Gotz, M. Ichimura, R. A. :oglia and A. Winther, Phys. Repts. 16C, 115 (1975).

15. N. Hashimoto, Progr. Theor. Phys. 59, 804 (1978).

16. R. Schaeffer and G. F. Bertsch, Phys. Lett. 38B, 159 (1972).

17. W. T. Pinkston and G. R. Satchler, Nucl. Phys. A (1982).

18. Y. Aoki, H. Iida, S. Kunori, K. Nagano, Y. Toba and K. Yagi, Phys. Rev. C 25, 1050 (1982).

19. G. R. Satchler, Direct Nuclear Reactions, Oxford University Press, Oxford, 1982 .

20. For example, see R. A. Broglia, 0. Hansen and C. Riedel, Adv. Nucl. Phys. 6, 287 (1973).

21. M. A. Nagarajan, M. R. Strayer and M. F. Werby, Phys. Lett. 67B, 141 (1977).

22. W. W. Daehnick, M. J. Spisak, J. R. Comfort, H. Hufner and H. H. Duhm, Phys. Rev. Lett. 41, 639 (1978); W. W. Daehnick, M. J. Spisak and J. R. Comfort, Phys. Rev. C23, 1906 (1981).

23. R. J. Peterson, R. E. Anderson and M. J. Fritts, Zeit. Phys. A302, 63 (1981). 